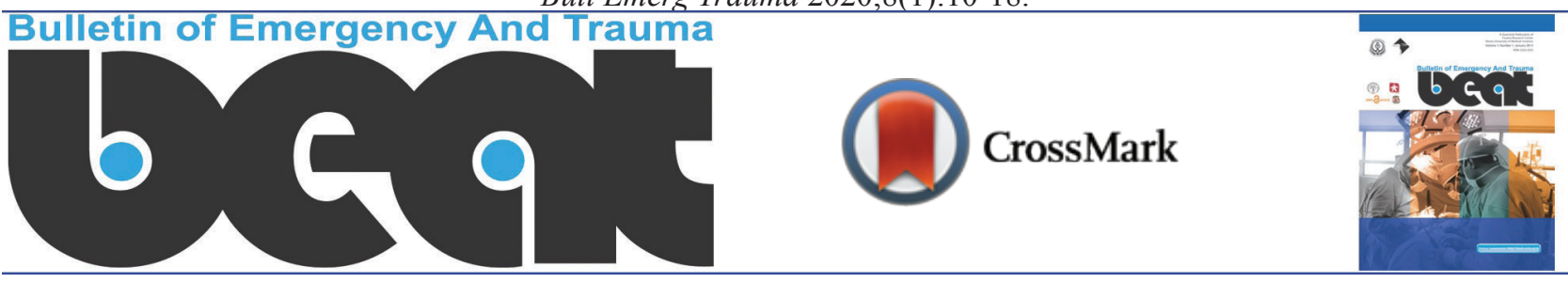

\title{
Regenerative Capacities of Chitosan-Nanoselenium Conduit on Transected Sciatic Nerve in Diabetic Rats: An Animal Model Study
}

\author{
Darab Faraji ${ }^{1}$, Mohsen Ebrahimi ${ }^{2 *}$, Babak Paknezhad ${ }^{2}$, Zahra Hami ${ }^{2}$, Alireza Jahandideh ${ }^{3}$
}

${ }^{1}$ Faculty of Medicine, AJA University of Medical Sciences, Tehran, Iran

${ }^{2}$ Department of Toxicology and Pharmacology, Faculty of Medicine, AJA University of Medical Sciences, Tehran, Iran

${ }^{3}$ Department of Clinical Science, Faculty of Specialized Veterinary Sciences, Science and Research Branch, Islamic Azad University, Tehran, Iran

*Corresponding author: Mohsen Ebrahimi

Address: Department of Toxicology and Pharmacology, Faculty of Medicine, AJA

University of Medical Sciences, Tehran, Iran.

e-mail: mepharmd@yahoo.com
Received: July 7, 2019

Revised: September 10, 2019

Accepted: October 10, 2019

\section{ABSTRACT}

Objective: To assess regenerative capacities of chitosan-nanoselenium conduit on transected sciatic nerve in diabetic rats.

Methods: A 10-mm sciatic nerve defect was bridged using a chitosan-nanoselenium conduit filled with phosphate buffered saline. In chitosan group, the chitosan conduit was filled with phosphate buffered saline solution. In sham-operated group, sciatic nerve was exposed and closed. In transected group, right sciatic nerve was transected and nerve cut ends were fixed in the adjacent muscle. The regenerated fibers were studied within 12 weeks after surgery.

Results: The behavioral and functional and electrophysiological tests confirmed faster recovery of the regenerated axons in chitosan-nanoselenium conduit group compared to chitosan group $(p=0.001)$. The mean ratios of gastrocnemius muscles weight were measured. There was statistically significant difference between the muscle weight ratios of chitosan-nanoselenium conduit and chitosan groups $(p=0.001)$. Morphometric indices of regenerated fibers showed number and diameter of the myelinated fibers were significantly higher in chitosan-nanoselenium conduit group than in chitosan group.

Conclusion: chitosan-nanoselenium conduit resulted in acceleration of functional recovery and quantitative morphometric indices of sciatic nerve.

Keywords: Peripheral nerve repair; Sciatic; Chitosan-nanoselenium conduit; Local.

Please cite this paper as:

Faraji D, Ebrahimi M, Paknezhad B, Hami Z, Jahandideh AR. Regenerative Capacities of Chitosan-Nanoselenium Conduit on Transected Sciatic Nerve in Diabetic Rats: An Animal Model Study. Bull Emerg Trauma. 2020;8(1):10-18. doi: 10.29252/beat-080103. 


\section{Introduction}

L ots of patients suffered from traumatic peripheral nerve injuries every year [1]. A considerable proportion of these patents were young people who constituted the main labor force of the society [2]. The nerve injuries caused the extremities dysfunction and affected their work and living abilities, which brought the society with a huge burden. The functional recovery of peripheral nerve injuries is associated with many factors, including the time interval between the injury and repair surgery [3].

However, some patients could not be treated with the nerve injuries such as the presence of multiple fractures requiring aggressive and expeditious management in preference to the nerve injuries [3]. The delayed nerve injuries treatment resulted in poor outcome of the extremity sensory and motor functions [4]. Studies in the control of diabetes mellitus and diabetic neuropathy [5] have renewed the interest in the rate and quality of nerve regeneration in this chronic disease. Although measurable improvements may follow better control of blood sugar and administration of aldose reductase inhibitors, complete recovery is dependent on the regeneration of damaged axons and the reestablishment of fully functional connection with their targets [6].

Several nerve guidance conduits (NGCs) and nerve protectant wraps are approved by the US Food and Drug Administration (FDA) for clinical use in peripheral nerve repair. These devices cover a wide range of natural and synthetic materials, which may or may not be resorbable [7]. Biodegradable nerve guides as a temporary scaffold are better than nondegradable biomaterials because the latter remain in situ as a foreign body and ultimately result in limiting recovery of nerve function [8].

Nevertheless, the resistance to biodegradation can be a cause of chronic nerve compression in the long run and a second surgery may therefore be required for its removal. Beneficial effects of chitosan as a conduit in promoting nerve regeneration have already been documented and it seems chitosan as a natural polymer has excellent properties including biocompatibility, biodegradability, nontoxicity and adsorption properties, and might be a suitable functional material for peripheral nerve regeneration $[9,10]$.

Selenium is one of the essential trace elements for humans. The bioavailability of selenium is related to its different chemical species. Recently, elemental selenium nanoparticles are attracting more and more attention due to their excellent high biological activity and lower toxicity [11]. Elemental selenium nanoparticles in liquid phase can be used as the materials for medical purposes [12]. For these applications, it is important to have good stability of elemental selenium nanoparticles in liquid phase [13].

One of the effective methods for stability of nanoparticles in liquid phase is to add modifiers.
Others used the chitosan as modifiers for the fabrication of elemental selenium nanoparticles [13]. Because of absence of available data on beneficial effects of nanoselenium on peripheral nerve regeneration, the present animal model study was conducted to assess regenerative capacities of chitosan-nanoselenium conduit on transected sciatic nerve in diabetic rats.

\section{Materials and Methods}

\section{Study Design}

Sixty male White Wistar rats weighing approximately $280 \mathrm{~g}$ were randomly divided into four experimental groups $(n=15)$ including transected control group, sham-operated group, a chitosan conduit group and a chitosan-nanoselenium conduit group. Each group was again subdivided into three subgroups of five animals each and surveyed within 12 weeks. Two weeks before and during the entire experiments, the animals were housed in individual plastic cages with an ambient temperature of $23 \pm 3^{\circ} \mathrm{C}$, stable air humidity, and a natural light/ dark cycle. The rats had free access to standard rodent laboratory food and tap water. All protocols were based working with laboratory animals of Iran Veterinary Organization.

\section{Preparation of Chitosan- Nano Selenium Biodegradable Matrix}

Water-soluble chitosan solution was prepared using a method described by others [14]. Briefly, Medium molecular weight crab shell chitosan was dissolved ( $\sim 00 \mathrm{kDa}, 85 \%$ deacetylated, Sigma- Aldrich St. Louis, MO, USA) into an aqueous solution (1\% $\mathrm{v} / \mathrm{v}$ ) of glacial acetic acid (Merck, Germany) to a concentration of $2 \%(\mathrm{w} / \mathrm{v})$, while stirring on a magnetic stirrer-hot plate. The solution was stirred with low heat $\left(50^{\circ} \mathrm{C}\right)$ for 3 hours. The resultant chitosan solution was filtered through Whatman filter paper after vacuum filtration to remove any un-dissolved particles.

For the preparation of elemental selenium nanoparticle sol, $1.5 \mathrm{~mL} 0.227 \mathrm{~mol} / \mathrm{L} \mathrm{Vc}$ was mixed with $1.0 \mathrm{~mL} 2.40 \mathrm{~mol} / \mathrm{L}$ acetum, then the appropriate amounts of $5.36 \mathrm{mmol} / \mathrm{L} \mathrm{Se}$ (IV) solution was added into the mixtures, the mixed solution was diluted to $10 \mathrm{~mL}$. For the preparation of selenium nanoparticlechitosan solution, appropriate amounts of chitosan solution were mixed with $1.5 \mathrm{~mL} 0.227 \mathrm{~mol} / \mathrm{L} \mathrm{Vc}$ and $1.0 \mathrm{~mL} 2.40 \mathrm{~mol} / \mathrm{L}$ acetum, respectively.

The appropriate amounts of $5.36 \mathrm{mmol} / \mathrm{L}$ Se (IV) solution was added into the mixtures, then the mixed solution was all diluted to $10 \mathrm{~mL}$. Philips diffractometer was used to obtain X-ray diffraction pattern. X-ray diffraction (XRD) patterns were acquired from $2 \theta=10^{\circ}$ to $80^{\circ}$ using $\mathrm{Cu} \mathrm{K \alpha}$ l radiation. Transmission electron microscope (Philips ES 30 KW0) was used to determine the size of nanoparticles (Figure 1). 


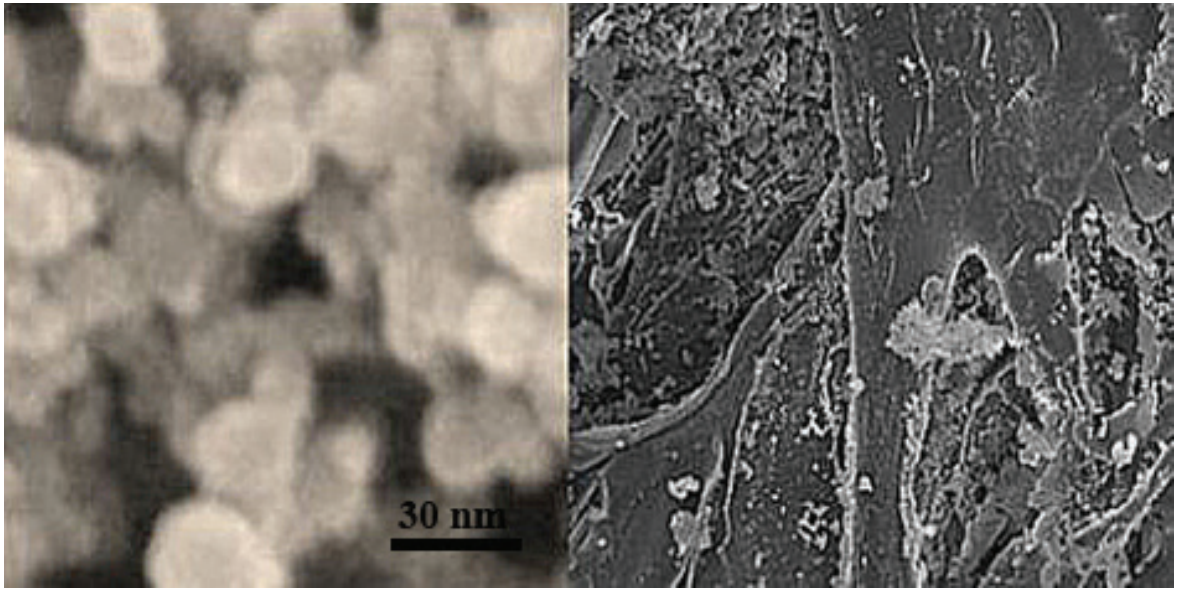

Fig. 1. (A) TEM micrograph of selenmium nano particles confirmed that nanoselenium nanoparticles were observed almost sphere like in morphology and approximately $30 \mathrm{~nm}$ in diameter. (B) SEM micrograph of chitosan-nanoselenium conduit.

\section{Surgery}

Animals were anesthetized by intraperitoneal administration of ketamine-xylazine (ketamine hydrochloride $5 \%, 90 \mathrm{mg} / \mathrm{kg}$ and xylazine hydrochloride $2 \%, 5 \mathrm{mg} / \mathrm{kg}$ ). The procedures were carried out based on the guidelines of the Ethics Committee of the International Association for the Study of pain [15]. The University Research Council approved all experiments.

Following surgical preparation in the shamoperated group, the right sciatic nerve was exposed through a gluteal muscle incision and after careful homeostasis the muscle was sutured with resorbable 4/0 sutures, and the skin was closed with $3 / 0$ nylon.

In transected control group, the right sciatic nerve was transected proximal to the tibio-peroneal bifurcation, where a $7 \mathrm{~mm}$ segment was excised, leaving a $10 \mathrm{~mm}$ gap due to retraction of nerve ends. Proximal and distal stumps were fixed in the adjacent muscle with 10/0 nylon epineurial suture. No graft was interposed between the stumps. In the chitosan group, the right sciatic nerve was exposed through a gluteal muscle incision and transected proximal to the tibio-peroneal bifurcation where a $7 \mathrm{~mm}$ segment was excised, leaving a gap about $10 \mathrm{~mm}$ due to retraction of nerve ends.

Proximal and distal stumps were each inserted $2 \mathrm{~mm}$ into a chitosan conduit and two 10/0 nylon sutures were placed at each end of the cuff to fix the tube in place and to leave a $10-\mathrm{mm}$ gap between the stumps. The conduit was filled with $20 \mu \mathrm{L}$ phosphate buffered saline. In the chitosan-nanoselenium conduit group, the chitosan-nanoselenium conduit was filled with phosphate buffered saline. The sterile vaseline was used to seal the ends of the tubes to avoid leakage. Currently acceptable technique of euthanasia in neurosciences include perfusion fixation. The animals were similarly anesthetized and euthanized with transcardial perfusion of a fixative containing $2 \%$ paraformaldehyde and $1 \%$ glutaraldehyde buffer ( $\mathrm{pH}=7.4) 4,8$ and 12 weeks after surgery.

\section{Behavioral Test}

Functional recovery of the nerve was assessed using the Basso, Beattie, and Bresnahan (BBB) locomotor rating scale for rat hind limb motor function [16]. Although BBB was widely used to assess functional recovery in spinal cord injured animals; however, it has been demonstrated that it could be most useful in assessment of never repair processes in peripheral nerve injuries [17]. The testing was performed in a serene environment. The animals were observed and assessed within a course of a 4-minute exposure to an open area of a mental circular enclosure. BBB scores were recorded once before surgery in order to establish a baseline control and again weekly thereafter to assess functional recovery during 12 weeks.

\section{Functional Assessment of Re-Innervation-Sciatic Functional Index (SFI)}

Walking track analysis was performed 4, 8 and 12 weeks after surgery based on others [18]. The lengths of the third toe to its heel (PL), the first to the fifth toe (TS), and the second toe to the fourth toe (IT) were measured on the experimental side $(\mathrm{E})$ and the contralateral normal side $(\mathrm{N})$ in each rat. The SFI in each animal was calculated by the following formula as $\mathrm{SFI}=-38.3 \times($ EPL-NPL $) / \mathrm{NPL}+109.5 \times($ ETS-NTS $) /$ NTS+13.3×(EIT-NIT)/NIT-8.8.

\section{Electrophysiological Studies}

After 12 weeks following the track test, all animals were subjected to electrophysiological studies using Nacro bio system (320-3760 A trace 80 , USA). Under general anesthesia, the left sciatic nerve was re-exposed by incision of the skin at the previous surgical site. Single electrical pulses at supra-maximal intensity were delivered via bipolar electrodes placed in turn at the proximal and distal trunk of the regenerated nerve. Electromyography (EMG) was recorded by inserting an electrode into the belly of gastrocnemius muscle.

The latency and the amplitude of EMG were obtained. Also, the difference in latency of EMG was measured, and the distance between the proximal 
and distal sites of stimulation was measured to calculate the conduction velocity across the regenerated nerve. On the contralateral, right intact side of each animal, similar measurements were made for the determination of conduction velocity. The conduction velocity of the bridged nerve was expressed as a percentage of that on the intact side of each animal to cancel off variations between animals (\% CVR). The recovery index of EMG amplitude in all groups was calculated based on Suzuki et al. using the following formula: Recovery index=Peak amplitude of the operated side/Peak amplitude of the intact side [19].

\section{Biomechanical Tests}

The regenerated nerves were harvested and placed in a normal saline bath at room temperature. The samples were then fixed between frozen fixtures in a mechanical apparatus. The TA.XTPlus Texture Analyzer mechanical test device was used for the assessment (Stable Micro Systems, Surrey GU7 $1 Y L, U K)$. After 5 minutes, the frozen fixtures were tightened to ensure that no slippage occurred during testing. The initial length was set to $10 \mathrm{~mm}$. Each sample was stretched at a constant rate of $1 \mathrm{~mm} / \mathrm{min}$. The load and displacement were sampled 5 times per second. Each sample was stretched to complete tensile failure. Samples were kept wet moist during testing using a drop of normal saline solution to the nerve segments.

\section{Muscle Mass}

Recovery assessment was also indexed using the weight ratio of the gastrocnemius muscles, 12 weeks after surgery. Immediately after sacrificing of animals, gastrocnemius muscles were dissected and harvested carefully from intact and injured sides and weighed while still wet, using an electronic balance. All measurements were made by two independent observers unaware of the analyzed group.

\section{Osmium Tetroxide Staining and Quantitative Morphometric Studies}

Four and 8 weeks after the $2^{\text {nd }}$ surgery, 6 rats in each group were deeply anesthetized with $10 \%$ chloral hydrate $(3.5 \mathrm{ml} / \mathrm{kg}$ weight intraperitoneally) and perfused through the left ventricle. Totally, 250 $\mathrm{ml}$ of warm saline flush was followed by $250 \mathrm{ml}$ of ice-cold 4\% paraformaldehyde in $0.1 \mathrm{M}, \mathrm{pH} 7.4$ phosphate buffer. After perfusion, the entire nerve, including the $1 \mathrm{~cm}$ from the suture proximally and $2 \mathrm{~cm}$ distally, was removed from each rat. One centimeter nerve segment proximal to the bifurcation was harvested and fixed in $4 \%$ paraformaldehyde in $0.1 \mathrm{M}$ phosphate buffer for 24 hours at 4 .

It was later stained in $1 \%$ osmium tetroxide for 12 hours and then dehydrated through a graded series of ethanols, and specimens were then immersed in xylene, embedded in paraffin, and sliced into $2-\mu \mathrm{m}$ cross-sections. Images were acquired under light microscopy (Olympus, Tokyo, Japan), from which the total number of myelinated axons and myelin thicknesses and diameters were evaluated. Morphometric measurements were performed using Image-pro Plus 5.0 software (Media Cybernetics). The shortest lengths of the outer and inner margins of the myelin sheath were measured to determine the fiber diameter and axon diameter. Myelin thickness was calculated after the fiber and axon diameters were determined.

\section{Statistical Analysis}

Experimental results were expressed as means \pm SD. Statistical analyses were performed using PASW 18.0 (SPSS Inc., Chicago, IL, USA). Model assumptions were evaluated by examining the residual plot. Results were analyzed using a factorial ANOVA with two between-subject's factors. Bonferroni test for pairwise comparisons was used to determine the effect of time and treatments. The differences were considered significant when $p<0.05$.

\section{Results}

\section{$B B B$ Recovery}

In order to assess hind limb recovery, the open field locomotor was used. Figure 2 shows BBB scores compared to the baseline. All groups, except for sham-operated group, showed the greatest degree of functional deficit one week after surgery. The chitosan-nanoselenium conduit group showed significant improvement in locomotion of the operated limb compared to the chitosan group during the study period $(p=0.001)$.

\section{Recovery of Sciatic Nerve Function and Reinnervation-SFI Outcome}

Figure 3 shows SFI values in experimental groups. Prior to surgery, SFI values in all groups were near

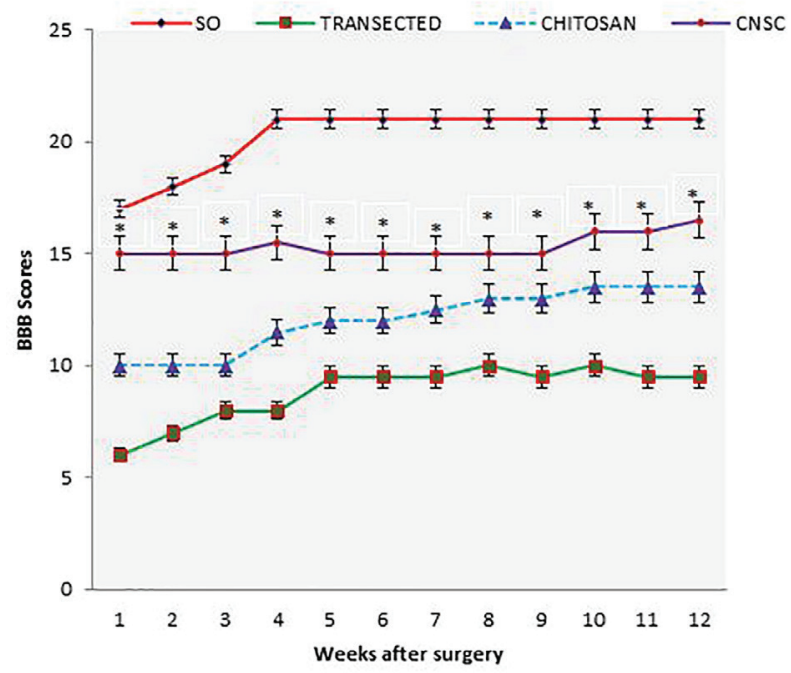

Fig. 2. BBB score for all experimental groups. Chitosannanoselenium conduit grafting gave better scores than in chitosan group. Standard error at each data point is shown with bars. 


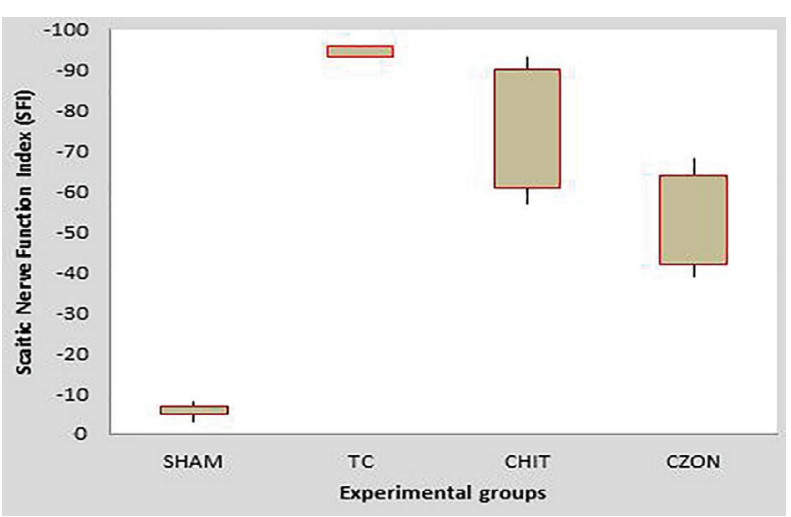

Fig. 3. Box-and-whisker plots of sciatic nerve function index values in each experimental group during the study period. Chitosan-nanoselenium conduit grafting gave better results in functional recovery of the sciatic nerve than in chitosan group.

zero. After the nerve transection, the mean SFI decreased to -100 due to the complete loss of sciatic nerve function in all animals. The statistical analyses revealed that the recovery of nerve function was significantly faster in chitosan-nanoselenium conduit group than in chitosan group $(p=0.001)$.

\section{Findings of Electrophysiological Studies}

Figure 4 and 5 show nerve conduction velocity (NCV) along regenerated sciatic nerves in experimental groups. NCV in chitosan-nanoselenium conduit group was significantly higher than that in chitosan group $(p=0.001)$.

\section{Biomechanical Measurements}

$\mathrm{F}_{\max }$ of nerve samples in experimental groups were shown in Table 1. $\mathrm{F}_{\max }$ in chitosan-nanoselenium conduit group was significantly higher than that in chitosan group $(p=0.001)$. Tensile strength, the amount of force per unit of initial cross-sectional area at tensile failure, was measured based on $\mathrm{F}_{\text {max }}$ and nerve cross sectional area. 12-week assessment revealed tensile strength of regenerated nerves treated with ibuprofen was higher than those in chitosan group ( $p=0.001)$. Ultimate strain, the amount of elongation divided by the initial specimen length achieved at the point of tensile failure, in chitosan-

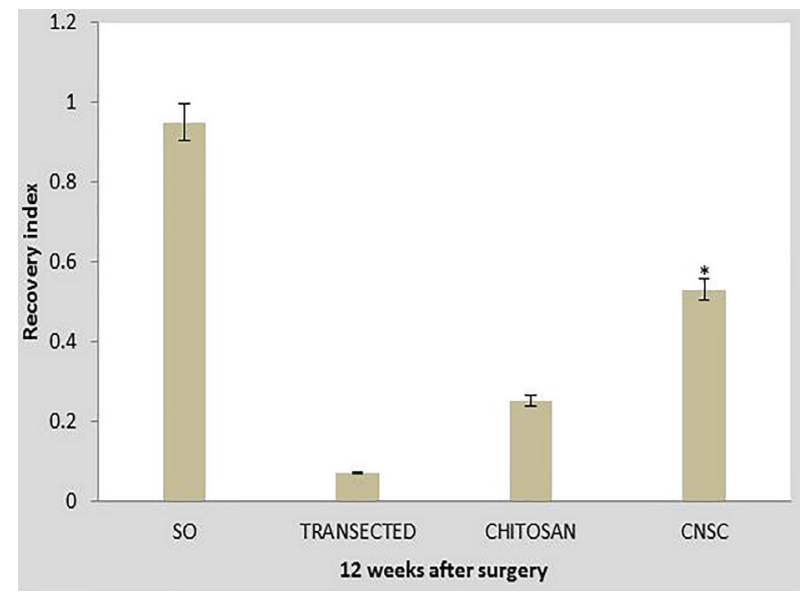

Fig. 5. Recovery index in experimental groups. Data are presented as mean \pm SD. $* P=0.001 v s$ chitosan group.

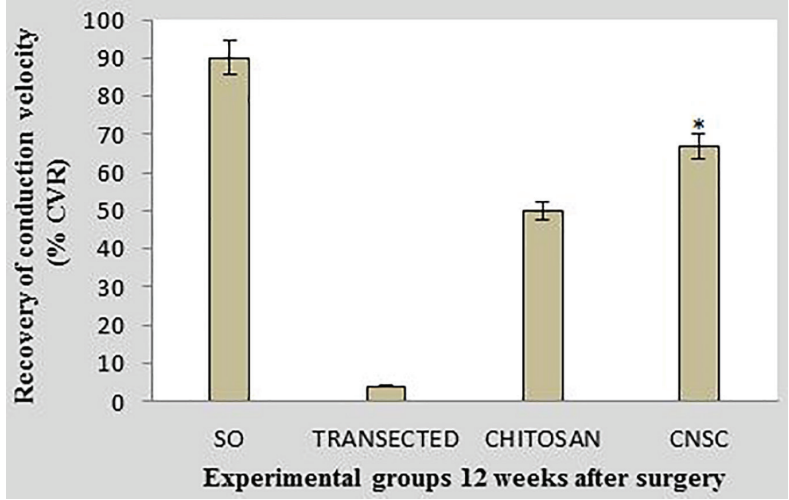

Fig. 4. Percentage recovery of conduction velocity in experimental groups. Data are presented as mean \pm SD. $* P=0.001 v s$ chitosan group.

nanoselenium conduit group was significantly higher than that in chitosan group ( $p=0.001)$. Toughness, reflecting the properties of anti-deformation and antifracture of the nerve, was determined by the nerve itself and could reflect "looseness" or "toughness" of the nerve. Toughness in chitosan-nanoselenium conduit group was significantly higher than that in chitosan group $(p=0.001)$.

\section{Muscle Mass Measurement}

Gastrocnemius muscles weight of injured and uninjured sides were measured in each group. There was statistically significant difference between percentage of the mean muscle weight ratios of chitosan-nanoselenium conduit and chitosan groups $(p=0.001)$. The findings showed that in chitosannanoselenium conduit group, the muscle weight ratio was bigger than in chitosan group (Figure 6).

\section{Morphometric Findings}

Table 2 shows quantitative morphometric analyses of regenerated nerves for each of the experimental groups. Four weeks after surgery, chitosannanoselenium conduit group presented significantly greater nerve fiber, axon diameter and myelin sheath thickness compared to chitosan group $(p=0.001)$. Although chitosan group presented regeneration

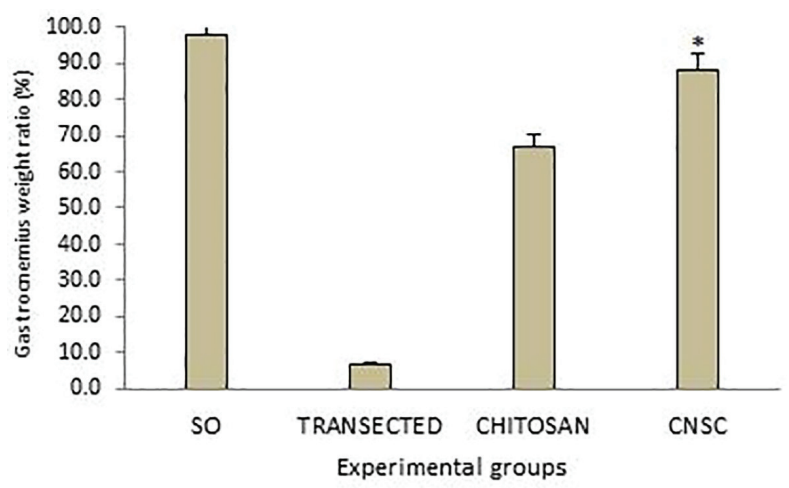

Fig. 6. Gastrocnemius muscle weight measurement. The gastrocnemius muscles of both sides (operated right and unoperated right) were excised and weighed in the experimental groups at 12 weeks after surgery. Data are presented as mean $\pm \mathrm{SD}$. $* \mathrm{P}=0.001$ vs chitosan group. 
Table 1. Biomechanical analyses of sciatic nerve in each of the experimental groups: Values are given as mean \pm SD

\begin{tabular}{lllll}
\hline Groups & $\begin{array}{l}\text { Tensile strength } \\
(\mathbf{M P a})\end{array}$ & $\begin{array}{l}\text { Maximum pull force } \\
(\mathbf{N})\end{array}$ & $\begin{array}{l}\text { Toughness } \\
(\mathbf{N} / \mathbf{m m})\end{array}$ & Ultimate strain \\
\hline Sham-operated & $7.30 \pm 1.20$ & $5.40 \pm 0.40$ & $1.25 \pm 0.55$ & $0.53 \pm 0.09$ \\
Transected & $2.33 \pm 0.30$ & $1.40 \pm 0.15$ & $0.23 \pm 0.08$ & $0.13 \pm 0.07$ \\
Chitosan & $3.10 \pm 0.13$ & $3.17 \pm 0.30$ & $0.43 \pm 0.20$ & $0.23 \pm 0.04$ \\
Chitosan-nanoselenium conduit & $4.37 \pm 0.15^{\mathrm{a}}$ & $4.34 \pm 0.24^{\mathrm{a}}$ & $0.78 \pm 0.10^{\mathrm{a}}$ & $0.38 \pm 0.05^{\mathrm{a}}$ \\
\hline
\end{tabular}

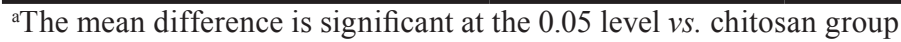

Table 2. Morphometric analyses of sciatic nerve in each of the experimental groups: Values are given as mean $\pm \mathrm{SD}$

\begin{tabular}{llll}
\hline Groups & $\begin{array}{l}\text { Myelin sheath thickness } \\
(\boldsymbol{\mu m})\end{array}$ & $\begin{array}{l}\text { Axon diameter } \\
(\boldsymbol{\mu m})\end{array}$ & $\begin{array}{l}\text { Axon counts } \\
\mathbf{f b} / \mathbf{m m}^{\mathbf{2}}\end{array}$ \\
\hline Sham-operated & $2.65 \pm 0.05$ & $11.35 \pm 0.15$ & $29068 \pm 2104$ \\
Transected & $1.05 \pm 0.03$ & $3.35 \pm 0.13$ & $4103 \pm 2103$ \\
Chitosan & $1.04 \pm 0.03$ & $3.70 \pm 0.12$ & $21347 \pm 2391$ \\
Chitosan-nanoselenium conduit & $1.04 \pm 0.07$ & $6.44 \pm 0.15^{\mathrm{a}}$ & $23172 \pm 2102^{\mathrm{a}}$ \\
\hline
\end{tabular}

${ }^{a}$ The mean difference is significant at the 0.05 level $v s$. chitosan group

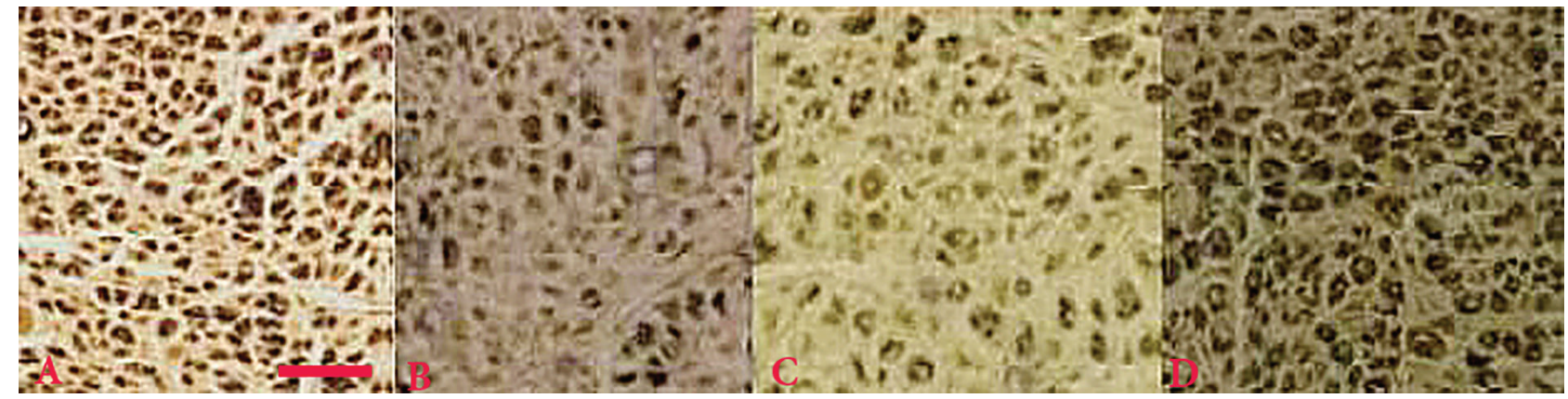

Fig. 7. Osmium staining of distal nerve stumps of the regenerated nerves 12 weeks after surgery from middle cable (A) sham-operated, (B) transected, (C) chitosan and (D) chitosan-nanoselenium conduit groups. Scale bar: $30 \mu \mathrm{m}$.

patterns, the morphometric indices in chitosannanoselenium conduit group, both after 8 and 12 weeks were better than in chitosan (Figure 7).

Using Factorial ANOVA analysis with two between-subject's factors (Group $\times$ time); in the chitosan-nanoselenium conduit group, the number of nerve fibers and myelin thickness did not show significant difference between 8 and 12 weeks intervals $(p=0.001)$. Mean thickness of myelin sheath from week 8 onward did not show significant difference between chitosan-nanoselenium conduit group and chitosan group $(p=0.001)$.

\section{Discussion}

Peripheral nerve injuries accounts for a considerable part of traumatic injuries around the world, especially in vehicle accidents and fall from a height. Such patients usually have other coalescent sufferings which must be treated in preference. So the nerve repair surgery would be postponed till the major injuries were controlled. In such condition, the neuronal body cannot receive the neurotrophic factors from the end organ in terms of retrograde axonal transport and die in consequences [20].

The nerve fibers degenerate and the muscle atrophies [21]. Poor outcome from peripheral nerve injury is especially evident when repair is delayed [22]. In order to improve the poor functional outcome of delayed nerve repair, some studies proposed effective methods to promote axonal regeneration. Nerve conduction measurement is a direct evidence for the study of nerve transmission [23]. The conduction velocity depends on the diameter of axons and the thickness of myelin sheath [24].

The results of the present study showed significantly different conduction velocity between the ibuprofen treated animals and eggsell membrane (ESM) bridged regenerated sciatic nerves, therefore, the ESM conduit in combination with ibuprofen could be assumed as a safe technique with no nerve conduction interference. The strongest connective tissue layers in peripheral nerves are the perineurium and, to a lesser extent, the epineurium. Changes in the epineurium and perineurium extracellular matrix composition are likely to have significant effects on the biomechanical properties of acellular nerve [25].

The connective tissue from the epineurium forms a layer of fiber membrane at the $3^{\text {rd }}$ day postoperatively and then forms collagen at the $8^{\text {th }}$ day. The key point influencing functional recovery is the number of axons throughout the suture that enhances the anti-tension capacity of the nerve [26]. Application of ibuprofen to regenerated nerve in the present study resulted in the enhanced biomechanical indices that were in agreement with functional and 
morphometric findings.

It is known from previous studies that regeneration process in rats would not have been completed by 12 weeks, a phenomenon which has been reported in a variety of experimental models [27]. Quantitatively, our results are consistent with these findings. However, a 12-week experimental period is sufficient for evaluation of regeneration process because in rats functional recovery after repair of a transected peripheral nerve occurs during this timeline [28].

The results of the present study showed that chitosan-nanoselenium conduit accelerated sciatic nerve functional recovery in diabetic rats. Nerve conduction measurement is a direct evidence for the study of nerve transmission [29]. The conduction velocity is dependent on the diameter of axons and the thickness of myelin sheath [24]. Our findings demonstrated that there was significantly different conduction velocity between cell treated animals and vein graft bridged regenerated sciatic nerves. Therefore, the chitosan conduit in combination with nanoselenium could be assumed as a safe nerve guide with no interference in nerve conduction.

Arrival of sprouts from the proximal stump at the distal nerve stump does not necessarily imply recovery of nerve function [28]. BBB is widely used to assess functional recovery in spinal cord injured animals, however, it has been demonstrated that it could be most useful in assessment of never repair processes in peripheral nerve injuries [30]. Results of the present study showed that chitosan-nanoselenium conduit treated animals were improved in locomotion of the operated limb compared to the control group during the study period.

Walking track analysis and static sciatic index has frequently been used to reliably determine functional recovery following nerve repair in rat models [31, 32]. It is a coordinated activity involving sensory input, motor response and cortical integration [28]. Recording wet muscle weight is a previously utilized alternative for motor target organ reinnervation [3335]. As the posterior tibial branch of the sciatic nerve regenerates into the gastrocnemius muscle, it will regain its mass proportional to the amount of axonal reinnervation [36, 37].

At the end of the present study, chitosannanoselenium conduit treated group showed significantly greater ratios of the mean gastrocnemius muscle weight in comparison to chitosan group indicating indirect evidence of successful end organ reinnervation. Quantitative morphometrical indices of regenerated nerve fibers showed significant difference between chitosan-nanoselenium conduit group and chitosan groups. Regarding better functional and morphometric indices in chitosan- nanoselenium conduit group versus chitosan group at the end of the study period, it could be stated that cell therapy, both accelerated and improved the process of nerve regeneration.

Despite much effort to introduce ideal therapeutic drugs for diabetic neuropathy, aldose reductase inhibitors have been shown to be the most established compounds among potent drugs. However, although experimental data on aldose reductase inhibitor have been very promising, their clinical efficacy seems limited even for mild degrees of diabetic neuropathy. Neurotrophic factors appear less effective than such conventional drugs; no extensive trials have shown their efficacy and a considerable number of adverse effects are also problematic [38].

Ongoing research in the field of electrically active nanomaterials includes the fabrication of composite materials with nanoscale, nanoselenium particles embedded into a polymer matrix. Nanoselenium, when mechanically deformed through ultrasound, for example, can theoretically provide an electrical stimulus, a known stimulatory cue for neural tissue regeneration. The combination of nanoscale surface dimensions and electrical activity may provide an enhanced neural tissue regeneration environment; such multifaceted nanotechnology approaches deserve further attention in the neural tissue regeneration field [39].

Even though our study showed the neuroregenerative action of chitosan-nanoselenium conduit in peripheral nerve injuries, data regarding the molecular mechanisms leading to the neuroprotective action remain to be investigated in depth. We have not given molecular evidence for neuroprotective action of chitosan-nanoselenium conduit. This may be considered as a limitation to our study.

In conclusion, this study demonstrated that chitosan-nanoselenium conduit improved functional recovery of transected sciatic nerve in rat. Supported by previous findings, the results from our present study would imply that the final outcome of both motor and sensory regeneration and reinnervation following repair of a peripheral nerve by chitosannanoselenium conduit may be of clinical benefit. There are reasonable grounds to believe that this approach could deliver a superior quality of reinnervation in a shorter period of time, compared to repair without nanoparticle treatment.

\section{Acknowledgments}

The authors would like to thank AJA University Deputy for Research, for the financial expertise.

Conflicts of Interest: None declared. 


\section{References}

1. Grinsell D, Keating CP. Peripheral nerve reconstruction after injury: a review of clinical and experimental therapies. Biomed Res Int. 2014;2014:698256.

2. Huckhagel T, Nüchtern J, Regelsberger J, Lefering R; TraumaRegister DGU. Nerve injury in severe trauma with upper extremity involvement: evaluation of 49,382 patients from the TraumaRegister DGU $®$ between 2002 and 2015. Scand J Trauma Resusc Emerg Med. 2018;26(1):76.

3. Jivan S, Kumar N, Wiberg M, Kay $\mathrm{S}$. The influence of pre-surgical delay on functional outcome after reconstruction of brachial plexus injuries. J Plast Reconstr Aesthet Surg. 2009;62(4):472-9.

4. Jonsson S, Wiberg R, McGrath AM, Novikov LN, Wiberg M, Novikova LN, et al. Effect of delayed peripheral nerve repair on nerve regeneration, Schwann cell function and targetmuscle recovery. PLoS One. 2013;(2):e56484.

5. Thomas PK, Eliasson SG. In Dyck PJ, Thomas PK, Lambert EH, Bunge RP. Peripheral Neuropathy, ed.; WB Saunders Co: Philadelphia; 1984.

6. Tomita K, Kubo $T$, Matsuda $K$, Fujiwara T, Yano K, Winograd JM, et al. The neurotrophin receptor p75NTR in Schwann cells is implicated in remyelination and motor recovery after peripheral nerve injury. Glia. 2007;55(11):1199-208.

7. Meek MF, Coert JH. US Food and Drug Administration/Conformit Europe- approved absorbable nerve conduits for clinical repair of peripheral and cranial nerves. Ann Plast Surg. 2008;60(4):466-72.

8. Jazayeri-Shooshtari SM, Namdar Z, Owji SM, Mehrabani D, MohammadiSamani S, Tanideh N, et al. Healing effect of lamotrigine on repair of damaged sciatic nerve in rabbit. $J$ Appl Anim Res. 2009;36(2):243-49.

9. Lin YL, Jen JC, Hsu SH, Chiu IM. Sciatic nerve repair by microgrooved nerve conduits made of chitosangold nanocomposites. Surg Neurol. 2008;70 Suppl 1:S1:9-18.

10. Wang G, Lu G, Ao Q, Gong Y, Zhang X. Preparation of crosslinked carboxymethyl chitosan for repairing sciatic nerve injury in rats Bio Technol Lett. 2010;32(1):59-66.

11. Wang HL, Zhang JS, Yu HQ. Elemental Selenium at Nano Size Possesses Lower Toxicity without Compromising the Fundamental Effect on Selenoenzymes: Comparison with Selenomethionine in Mice. Free Radical Biology and Medicine. 2007;42(10):1524-33.

12. Gao $X$, Zhang J, Zhang L. Hollow sphere selenium nanoparticles: their in-vitro anti hydroxyl radical effect. Advanced Materials. 2002;14(4):290-3.

13. Bai Y, Wang Y, Zhou Y, Li W, Zheng W. Modification and modulation of saccharides on elemental selenium nanoparticles in liquid phase. Materials letters. 2008;62(15):2311-4.

14. Ojagh SM, Rezaei M, Razavi SH, Hosseini SMH. Development and evaluation of a novel biodegradable film made from chitosan and cinnamon essential oil with low affinity toward water. Food Chemistry. 2010;122(1):161-6.

15. Zimmermann M. Ethical guidelines for investigations of experimental pain in conscious animals. Pain. 1983;16(2):109-10.

16. Basso DM, Beattie MS, Bresnahan JC. A sensitive and reliable locomotor rating scale for open field testing in rats. J Neurotrauma. 1995;12(1):1-21.

17. Dinh P, Hazel A, Palispis W, Suryadevara S, Gupta R. Functional assessment after sciatic nerve injury in a rat model. Microsurgery. 2009;29(8):644-9.

18. Bain JR, Mackinnon SE, Hunter DA. Functional evaluation of complete sciatic, peroneal, and posterior tibial nerve lesions in the rat. Plast Reconstr Surg. 1989;83(1):129-38.

19. Di Benedetto G, Zura G, Mazzucchelli R, Santinelli A, Scarpelli M, Bertani A. Nerve regeneration through a combined autologous conduit (vein plus acellular muscle grafts). Biomaterials. 1998;19(1-3):173-81.

20. Chowdary PD, Che DL, Cui B. Neurotrophin signaling via longdistance axonal transport. Annu Rev Phys Chem. 2012;63:571-94.

21. Rakei SM, Rahmanian A, Saffarian A, Shafeian R, Mehrabani D. Function recovery after transplantation of fetal brain tissue into injured spinal cord in experimental rats. $J$ Appl Anim Res. 2009;36(2):303-6.

22. Gashmardi N, Mehrabani D, Khodabandeh Z, Hosseini SM. Effect of bone marrow-derived mesenchymal stem cells on changes of serum levels of tnf- $\alpha$ and locomotor function after spinal cord injury in mice. $J$ Med Sci. 2016;16(1-2):16-24.

23. Matsumoto K, Ohnishi K, Kiyotani T, Sekine T, Ueda H, Nakamura T, et al. Peripheral nerve regeneration across an 80 -mm gap bridged by a polyglycolic acid (PGA)-collagen tube filled with laminin-coated collagen fibers: a histological and electrophysiological evaluation of regenerated nerves. Brain Res. 2000;868(2):315-288.

24. Chen CJ, Ou YC, Liao SL, Chen WY, Chen SY, Wu CW, et al. Transplantation of bone marrow stromal cells for peripheral nerve repair. Exper Neurol. 2007;204(1):443-53.

25. Ma XL, Sun XL, Yang Z, Li XL, Ma JX, Zhang Y, et al. Biomechanical properties of peripheral nerve after acellular treatment. Chin Med J (Engl). 2011;124(23):3925-9.

26. Jiang $B$, Zhang $P$, Yan J, Zhang $H$. Dynamic observation of biomechanic properties of sciatic nerve at the suture site in rats following repairing. Artif Cells Blood Substit Immobil Biotechnol. 2008;36(1):45-50.

27. Gattuso JM, Glasby MA, Gschmeissner SE, Norris RW. A comparison of immediate and delayed repair of peripheral nerves using freeze-thawed autologous skeletal muscle grafts - in the rat. Br J Plast Surg. 1989;42(3):306-13.

28. Castaneda F, Kinne RK. Omental graft improves functional recovery of transected peripheral nerve. Muscle Nerve. 2002;26(4):527-32.

29. Matsumoto K, Ohnishi K, Kiyotani T, Sekine T, Ueda H, Nakamura T, et al. Peripheral nerve regeneration across an $80-\mathrm{mm}$ gap bridged by a polyglycolic acid (PGA)-collagen tube filled with laminin-coated collagen fibers: a histological and electrophysiological evaluation of regenerated nerves. Brain Res. 2000;868(2):315-28.

30. Dinh P, Hazel A, Palispis W, Suryadevara S, Gupta R. Functional assessment after sciatic nerve injury in a rat model. Microsurgery. 2009;29(8):644-9.

31. Bain JR, Mackinnon SE, Hunter DA. Functional evaluation of complete sciatic, peroneal, and posterior tibial nerve lesions in the rat. Plast Reconstr Surg. 1989;83(1):129-38.

32. De Medinaceli L, Freed WJ, Wyatt RJ. An index of the functional condition of rat sciatic nerve based on measurements made from walking tracks. Exp Neurol. 1982;77(3):634-43.

33. Menderes A, Yilmaz M, Vayvada $H$, Ozer E, Barutçu A. Effects of nerve growth factor on the neurotization of denervated muscles. Ann Plast Surg. 2002;48(4):415-22.

34. Fitton A, Berry M, McGregor A. Preservation of denervated muscle form and function by clenbuterol 
in a rat model of peripheral nerve injury. The Journal of Hand Surgery: British \& European Volume. 2001;26(4):335-46.

35. Day CS, Riano F,Tomaino MM, et al. Growth factor may decrease muscle atrophy secondary to denervation. $J$ Reconstr Microsurg. 2001;17(1):51-7.

36. Hou Z, Zhu J. An experimental study about the incorrect electrophysiological evaluation following peripheral nerve injury and repair. Electromyogr Clin Neurophysiol. 1998;38(5):301-4.

37. Evans GR, Brandt K, Widmer MS, Lu L, Meszlenyi RK, Gupta PK, et al. In vivo evaluation of poly (L-lactic acid) porous conduits for peripheral nerve regeneration. Biomaterials. 1999;20(12):1109-15.
38. Yasuda H, Terada M, Maeda K, Kogawa S, Sanada M, Haneda $\mathrm{M}$, et al. Diabetic neuropathy and nerve regeneration. Progress in neurobiology. 2003;69(4):229-85.

39. Seil JT, Webster TJ. Electrically active nanomaterials as improved neural tissue regeneration scaffolds. Wiley Interdiscip Rev Nanomed Nanobiotechnol. 2010;2(6):635-47. and share. Bulletin of Emergency And Trauma articles are published under a Creative Commons license (CC-BY-NC). 